\title{
Hydra Molecular Network Reaches Criticality at the Symmetry-Breaking Axis-Defining Moment
}

\author{
Jordi Soriano, ${ }^{1}$ Cyril Colombo, ${ }^{2}$ and Albrecht Ott ${ }^{1, *}$ \\ ${ }^{1}$ Experimentalphysik I, Universität Bayreuth, D-95440 Bayreuth, Germany \\ ${ }^{2}$ Liquid Propulsion Department, EADS Space Transportation, F-78130 Les Muraux, France \\ (Received 24 April 2006; published 18 December 2006)
}

\begin{abstract}
We study biological, multicellular symmetry breaking on a hollow cell sphere as it occurs during hydra regeneration from a random cell aggregate. We show that even a weak temperature gradient directs the axis of the regenerating animal - but only if it is applied during the symmetry-breaking moment. We observe that the spatial distribution of the early expressed, head-specific gene $k s 1$ has become scale-free and fractal at that point. We suggest the self-organized critical state to reflect long range signaling, which is required for axis definition and arises from cell next-neighbor communication.
\end{abstract}

The hydra [Fig. 1(a)], a phylogenetically old organism, has become an attractive model organism in the hope to better understand the evolution of development [1]. A hydra consists of a cylindrical body column with a foot at one end and a head equipped with a mouth opening and tentacles at the other. An adult hydra is in a steady state: Cells are continuously multiplying at its center; with age, they migrate to both ends of the body column. Only when the cells reach either end do they become irreversibly specialized or "differentiated," and they are subsequently lost.

The hydra has astonishing regeneration capabilities [2]; it can even reform from a completely disordered cell ball, condensed from dissociated, single hydra cells [3]. Only cells from the body column contribute to regeneration; terminally differentiated cells are expelled during the process. During hydra regeneration, the remaining $10^{4}$ quasiidentical, multipotent cells need to break the initial developmental isotropy, define a single axis, and maintain it. Our study focuses on this symmetry-breaking aspect. Contrary to the hydra, most other organisms preserve an initial asymmetry during development and translate it to the axis of the animal [4]. Only in a very few cases were hydrodynamic flow or electric fields as external factors shown to direct the developmental symmetry [5].

Much effort has focused on the isolation and characterization of different hydra genes and signaling cascades [69]. Among the genes shown to play a role in early development is the hydra-specific gene $k s 1$ [10] and Wnt [11]. $k s 1$ is permanently expressed and upregulated in response to early signals of head formation. The graded expression of $k s 1$ along the body column of an adult hydra is regarded as characteristic for the decay in the head forming potential [12]. The Wnt gene is well known to have axis-related roles in many species. The hydra expresses $W n t$ in a few irreversibly differentiated cells located at the tip of the head, a region called the hypostome [Fig. 1(a)].

A common feature of multicellular embryogenesis is the formation of the so-called organizer [4], a cluster of the first few irreversibly differentiated cells, which subse- quently guides the fate of all of the others. In vertebrates, the occurrence of the (Spemann) organizer precedes gastrulation, the invagination of the embryonic cell ball, which initiates gut formation and reveals the animal axis. In hydra, the organizer is the first structure to be restored during regeneration [7] [Fig. 1(b)], with Wnt expressed in the organizer region only [11].

Child hypothesized in 1929 that a metabolic rate gradient should dictate the embryonic axis position [13]. Metabolic rates depend strongly on temperature - when taken as a simple activated process, activation energies are in the range of $(20-80) k T, T$ the temperature, $k$ the Boltzmann constant. Although numerous experiments on different model systems in a temperature gradient failed to confirm Child's hypothesis [14], we show in this Letter that even a weak temperature gradient dictates the axis of the developing hydra - as long as it is applied during the axislocking moment. We show that, in homogeneous temperature, $k s 1$ gene expression domains become self-similar close to this symmetry-breaking moment. We conclude
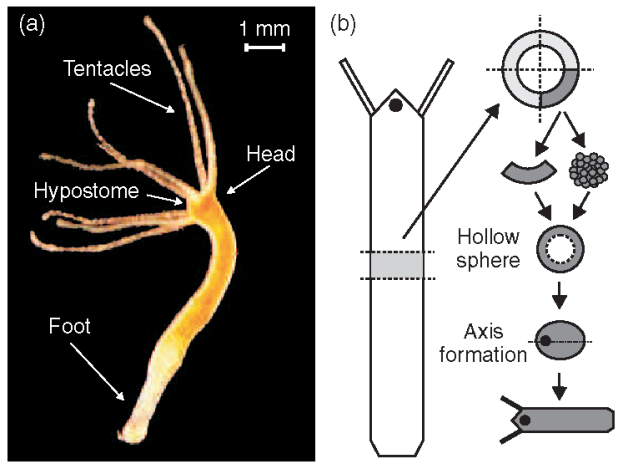

FIG. 1 (color online). (a) Adult Hydra vulgaris. (b) Sketch of the regeneration process. A small disk of tissue is excised from the body column of an adult hydra and cut into four fragments. Such a fragment, even when dissociated to single cells and reagreggated, forms a hollow sphere, breaks the symmetry with the formation of a new axis, and develops to an adult hydra. Black dots indicate the position of the organizer. 
that the hydra molecular network self-organizes to become critical before it breaks its symmetry spontaneously. We propose that the multipotent cells need to increase their fluctuations in the expression profile to create an asymmetry. The hydra locks this asymmetry as the fluctuations become scale-free and therefore reach system size. We suggest that the absence of scale is necessary to form exactly one axis. We discuss similarities with selforganized critical models.

Hydra biomechanical properties and the effect of a temperature gradient. - Hydra regeneration starts with the formation of a hollow sphere, made of a cell bilayer [Fig. 1(b)]. The subsequent development is accompanied by cycles of steady inflation and fast contraction, as shown in Fig. 2(a). We have described these oscillations as pressure buildup in the hydra ball due to osmotic swelling $[15,16]$ until a sudden pressure release occurs. The cycles change abruptly after about 20 hours from high amplitude, low frequency oscillations (phase I) to low amplitude, high frequency oscillations (phase II) [Fig. 2(a)]. The deflation mechanism of the hydra ball is different in both phases: During phase I, it is the rupture of the cell bilayer which causes cell ball deflation. During phase II, a weak spot in the hydra cell bilayer causes the sphere to release pressure

(a)
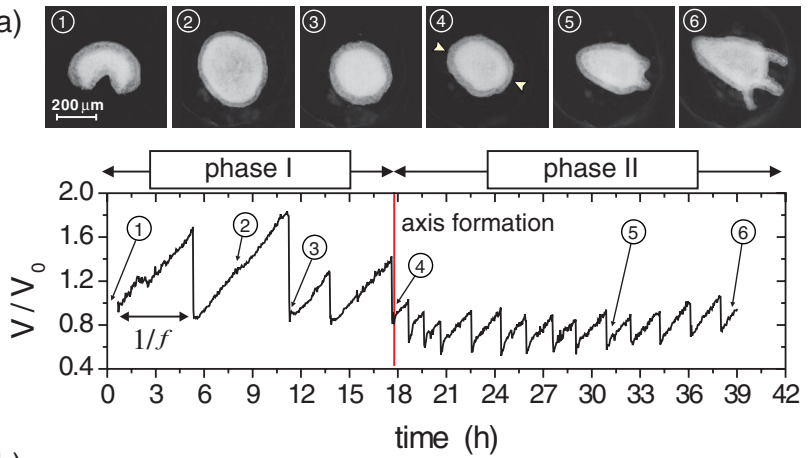

(b)

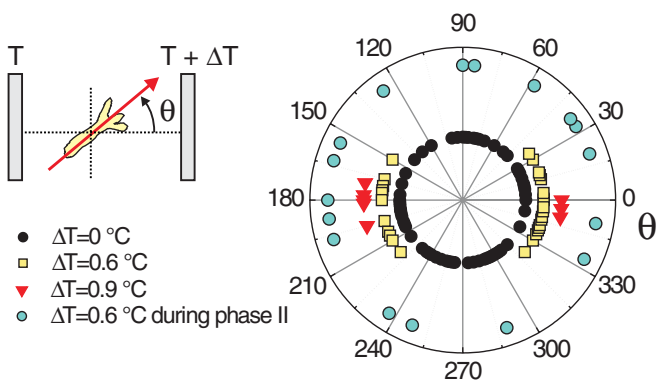

FIG. 2 (color online). (a) Mechanical oscillations during regeneration. Above: Snapshots of different regeneration stages of a small hydra fragment; arrows in 4 mark the future axis. Below: Corresponding volume oscillations. (b) Angular distribution of the hydra head orientation with respect to the direction of a temperature gradient $\left(0^{\circ}\right.$ : head forms at the hot side). The difference $\Delta T$ is across the cell aggregate $(300 \mu \mathrm{m})$. Triangles and squares: Temperature gradient applied from the beginning; shaded circles: $\Delta T=0.6^{\circ} \mathrm{C}$ applied during phase II only; black circles: control at constant temperature. at a decreased threshold, thus reducing the amplitude of the inflation-contraction cycles. At the transition between both phases, the elastic properties of the cell sphere become anisotropic in the direction of the developmental axis $[15,17]$.

We have observed that a hydra develops following a weak temperature gradient in all of the studied samples [Fig. 2(b)], regardless of whether the hydra sphere was made from a small fragment of hydra tissue or from reaggregated cells [17]. However, the axis of the animal forms along the temperature gradient only if the application of the gradient starts within phase I oscillations. Hydra spheres that have been put into the temperature gradient later, after a switch to small amplitude phase II oscillations, have an arbitrary axis position. The regeneration time of these spheres is diminished by the time span of the (lacking) phase I oscillations. The same applies for larger spheres made from fragments. They retain their original axis, lack phase I oscillations, and develop faster accordingly [17].

We conclude from these experiments that the axis orientation of the developing hydra sphere is irreversibly locked upon reduction of the amplitude of the inflationcontraction cycles. The axis, however, is undefined during phase I oscillations.

Ks 1 expression patterns. - RNA in situ hybridization labels the cells that express the gene of interest with a colored dye [Fig. 3(a)] [10,17] [for hydra, quantitative labeling of gene expression with green fluorescent protein is not yet routinely available [18]]. We study $k s 1$ expression patterns on the hydra sphere in homogeneous temperature. The pattern develops a characteristic size before and after symmetry breaking, but its distribution is scalefree after about 20 hours: When the symmetry-breaking moment is reached, it follows a power law [Fig. 3(b)]. We observe that the $k s 1$ expression pattern becomes fractal [Fig. 3(c)] at the same time. The fractal pattern is not completely lost during the following hydra shape change towards an increasingly oblong ellipsoid. A possible explanation is that the $k s 1$ expressing cells tend to maintain their expression profile at this point. Although scaling of gene expression has been observed before [19], our observation differs as we consider the size of patches of cells, which are "infected" with $k s 1$ in a basically twodimensional sheet of tissue.

Since the cells are identically programmed initially, the fact that disjoint groups of cells do or do not express $k s 1$ must be interpreted with fluctuations in gene expression. Already, Mitgutsch et al. observed strong fluctuations of the head generating expression pathways [20] including transient phases of head formation. Hydra gene expression fluctuates until it becomes scale-free and fractal in vicinity to the symmetry-breaking moment. This state is identified as critical since the system becomes susceptible to a weak perturbation, here in the form of a temperature gradient, 
(a)

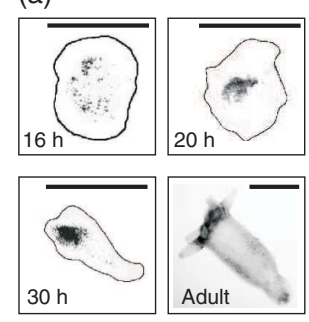

(c)

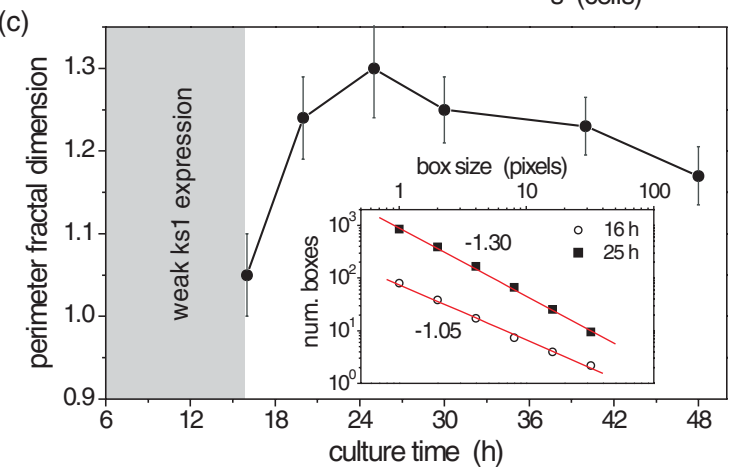

FIG. 3 (color online). (a) Examples of $k s 1$ patterns at different developmental stages. Scale bar, $500 \mu \mathrm{m}$. (b) $K s 1$ spot-size probability distribution for 3 different culture times. $P$ is the probability for a cell to be part of a spot larger than or equal to $s$ (cells) as a function of $s$, averaged over $6 \mathrm{ks} 1$ patterns. All curves start at $P=1$; vertical shift for clarity only; the line is a least squares fit. With $n(s) \sim s^{-\tau}$ the frequency of occurrence, $P \sim$ $s^{-(\tau-2)}$, leading to $\tau=2.8 \pm 0.1$ for $25 \mathrm{~h}$ [17]. (c) Perimeter fractal dimension from the beginning of expression $(\sim 16 \mathrm{~h})$ to regeneration, averaged over 6 patterns. Inset: Examples of log$\log$ fits to determine the perimeter fractal dimension.

which spatially directs the spontaneous symmetry breaking. Criticality is a self-organized state of the molecular network.

We observe that fluctuations reach system size at the axis-locking moment. However, $k s 1$ scaling with the experimentally observed exponents cannot have infinite reach (and the hydra does not reach the exact critical state) since the probability to observe an infinite cluster is given by $\lim _{l \rightarrow \infty} l^{2} \int_{s(l)}^{\infty} n(s) d s \sim l^{2}\left(l^{1.7}\right)^{(-2.8+1)}=0$ [where $l^{2}$ is the number of lattice elements, $s(l)$ the spanning cluster, $n(s) \sim s^{-2.8}$ from Fig. 3(b), and 1.7 is the area fractal dimension]. System-wide communication is necessary to avoid formation of multiple heads. Indeed, more than one head forms on randomly assembled regenerating hydra cell aggregates, which are substantially larger in size than in our experiments [11]. We postulate that the power law must break down at the typical distance between such heads. The size of our aggregates is such that it corresponds to the reach of the power law; this does not mean that the hydra necessarily detects its critical state.

Possible origin of molecular network criticality.-At present, there is no evidence for chemical long range communication in hydra; a large body of evidence points towards a major role of next-neighbor interactions between cells [21]. Zernike showed that in 2 or more dimensions on a next-neighbor lattice the information decay changes abruptly from an exponential (i.e., short range) to a power law (long range) with increasing exchange probability [22] due to increased branching. In the case of $n$ neighbors, the transition to scaling occurs if the exchange probability between neighboring sites reaches $1 / n$ [23,24]. Following this idea, scaling as we observe it in our study arises naturally as a signature of an increasingly "excited" state of the cell population.

Based on Zernike's idea, Bak et al. showed theoretically that self-organized critical (SOC) systems may result from next-neighbor interaction with simple rules [23]. We therefore ask if any of the SOC models, which are claimed to be very general, explains the SOC state of hydra. A SOC model that may come close to our situation is the forest fire model, connected to excitable media [23]. The model requires nonlinear next-neighbor communication and well separated time scales (fast burning, slow regrowth, very rare lightning). Biological cells are known to be nonlinear, and, in principle, our $k s 1$ expression data rather corroborate a separation of time scales. In 2 dimensions, forest fire models give exponents $\tau \simeq 2.1-2.2$ for the fire size distribution and a fractal area dimension $D \simeq 1.8-1.9$ [23] compared to our experimental values $\tau \simeq 2.8$ and $D \simeq 1.7$ (1.3 for the perimeter fractal dimension). The forest fire model depends on the lattice size and the details of the model rules [25]. The connectivity in the hydra cell-bilayer sheet is higher than on an ideal two-dimensional lattice. Gene expression is certainly noisy. As already shown, the hydra does not reach the critical state completely. We conclude that the agreement is more of a qualitative nature.

The SOC forest fire model was claimed to reflect the spreading of mumps and similar diseases in a closed population [26]. Mumps, as well as real forest fires [27], have similar exponents as in our experiment. Although highly optimized tolerance [27] predicted the exact exponent for the latter, it does not relate to a fractal state nor to fluctuation driven symmetry breaking as we observe it here. It is an interesting question whether a reaction-diffusion mechanism as in Ref. [28] could also be tuned to generate the observed gene expression patterns.

Wnt expression and axis locking. - As shown in Fig. 4, the number of $k s 1$ expressing cells increases during axis formation to reach a plateau after axis locking. The sharpest increase in $k s 1$ expression coincides with the transition to phase II motion [Fig. 2(a)], indicating that the sudden change in the mechanical motion correlates with early head formation. Hobmayer et al. [11] determined the time frame of Wnt expression. Both results together show that the axis-locking moment coincides with Wnt expression. Incorporation of Wnt expressing cells from the hypostome in cell aggregates shortens phase I oscillations considerably and defines the axis position [17]. Regenerating hyd- 


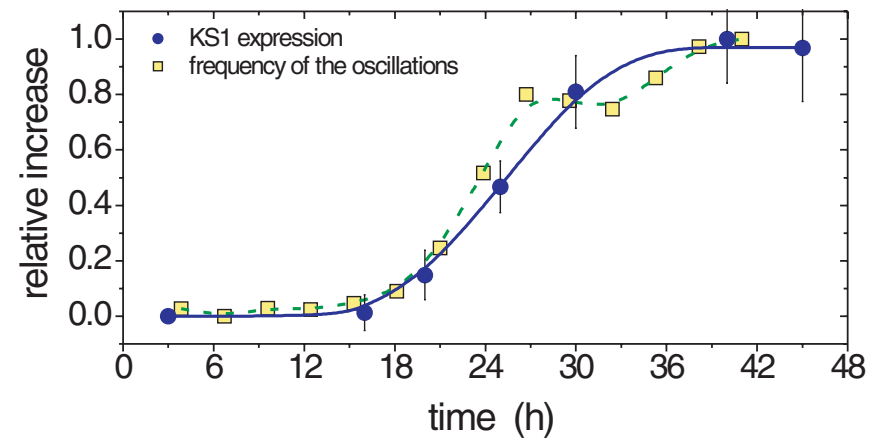

FIG. 4 (color online). Temporal evolution of the (normalized) fraction of $k s 1$ expressing cells (circles, each point an average over 6 patterns) compared with the relative increase of the frequency of the oscillations $\left(f-f_{\min }\right) / f_{\max }$ (squares, averaged over 18 evolving spheres), where $f_{\min }$ and $f_{\max }$ are, respectively, the minimum and maximum frequencies measured along the regeneration process. The lines are a guide to the eye.

ras that remain stuck accidentally in phase I oscillations do not show prominent signs of developmental asymmetry until they die after a few days.

There is increasing evidence of a molecular connection between $W n t$ and cell adhesion [29]. We suggest that it is a Wnt expressing organizer, consisting of only 5-10 cells, which causes the localized weakening of the cell bilayer and the release of osmotic pressure during phase II. This agrees with the expected size of the pressure-releasing hole, of the order of cell size [15]. Following this idea, the weakening of the cell bilayer can be understood as early mouth formation, a topological change providing a certain parallel to gastrulation.

In principle, each of the 10000 hydra cells can differentiate irreversibly into a member of an axis-organizing unit $[2,30]$, and fast global inhibition-reaching every cell - is needed in order to prevent the formation of a second axis. Long range signaling across cell membranes is difficult and slow. Sometimes cells use physical means such as hydrodynamic flow [5] to exchange information globally. One may speculate that hydra cells detect the sudden change in topology (early mouth formation) in order to transmit information about axis locking fast enough. It is clear that once one organizer is formed, it continues to slowly chemically suppress the formation of a secondary axis even for cells with a high $k s 1$ expression level, as known from the adult animal.

Conclusions. - Our experiments highlight a novel way of embryonic axis definition by spontaneous symmetry breaking through a critical mechanism. Controlled fluctuation driven symmetry breaking appears as unnecessarily complicated; however, it may have been important in evolution, when identical, single cells self-organized to form multicellular organisms.
We thank M. Kücken and P. A. Pullarkat for critical reading of the manuscript, A. Hanold for technical assistance, and T. C. G. Bosch (Kiel, Germany) for discussions. J.S. acknowledges the support of the European Training Network PHYNECS, No. HPRN-CT-2002-00312.

*Electronic address: albrecht.ott@uni-bayreuth.de

[1] B. Galliot and V. Schmid, Int. J. Dev. Biol. 46, 39 (2002).

[2] H. R. Bode, Dev. Dyn. 226, 225 (2003).

[3] U. Technau et al., Proc. Natl. Acad. Sci. U.S.A. 97, 12127 (2000).

[4] Wolpert Lewis, Principles of Development (Oxford University Press, New York, 1999).

[5] Y. Okada et al., Cell 121, 633 (2005).

[6] T. C. G. Bosch and K. Khalturin, Can. J. Zool. 80, 1670 (2002).

[7] R. E. Steele, Dev. Biol. 248, 199 (2002).

[8] T. W. Holstein et al., Dev. Dyn. 226, 257 (2003).

[9] T. Fujisawa, Dev. Dyn. 226, 182 (2003).

[10] R. Weinziger et al., Development (Cambridge, U.K.) 120, 2511 (1994).

[11] B. Hobmayer et al., Nature (London) 407, 186 (2000).

[12] I. Endl et al., Proc. Natl. Acad. Sci. U.S.A. 96, 1445 (1999).

[13] C. Child, Wilhelm Roux' Arch. Entwicklungsmech. Organ. 117, 21 (1929).

[14] S. Black, Dev. Biol. 134, 376 (1989).

[15] C. Fütterer et al., Europhys. Lett. 64, 137 (2003).

[16] E. Nicola et al. (to be published).

[17] See EPAPS Document No. E-PRLTAO-97-037651 for experimental details and data analysis. For more information on EPAPS, see http://www.aip.org/pubservs/epaps. html.

[18] J. Wittlieb et al., Proc. Natl. Acad. Sci. U.S.A. 103, 6208 (2006).

[19] C. Furusawa and K. Kaneko, Phys. Rev. Lett. 90, 088102 (2003); H. R. Ueda et al., Proc. Natl. Acad. Sci. U.S.A. 101, 3765 (2004).

[20] C. Mitgutsch et al., Dev. Biol. 207, 189 (1999).

[21] S. E. Fraser et al., Science 237, 49 (1987).

[22] F. Zernike, Physica (Amsterdam) 7, 565 (1940).

[23] H.J. Jensen, Self-Organized Criticality (Cambridge University Press, Cambridge, England, 1998).

[24] D. Sornette, Critical Phenomena in Natural Sciences (Springer, New York, 2004), 2nd ed.

[25] G. Pruessner and H. J. Jensen, Phys. Rev. E 65, 056707 (2002), and references therein.

[26] C. J. Rhodes and R. M. Anderson, Nature (London) 381, 600 (1996).

[27] J. M. Carlson and J. Doyle, Proc. Natl. Acad. Sci. U.S.A. 99, 2538 (2002), and references therein.

[28] A. Gierer and H. Meinhardt, Kybernetik 12, 30 (1972); H. Meinhardt, Curr. Opin. Genet. Dev. 14, 446 (2004).

[29] W. J. Nelson and R. Nusse, Science 303, 1483 (2004).

[30] M. Broun and H.R. Bode, Development (Cambridge, U.K.) 129, 875 (2002). 